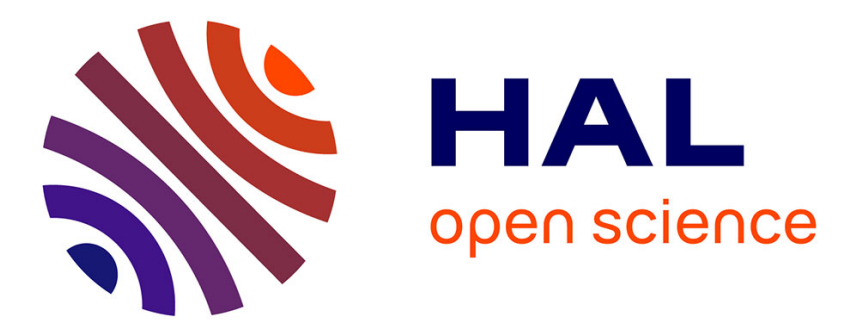

\title{
Effet d'une levée de dormance incomplète sur la qualité des étamines et du pollen chez le fraisier (Fragaria $\mathrm{x}$ ananassa Duch) \\ Georgette Risser
}

\section{- To cite this version:}

Georgette Risser. Effet d'une levée de dormance incomplète sur la qualité des étamines et du pollen chez le fraisier (Fragaria x ananassa Duch). Agronomie, 1985, 5 (9), pp.813-818. hal-00884816

\section{HAL Id: hal-00884816 https://hal.science/hal-00884816}

Submitted on 1 Jan 1985

HAL is a multi-disciplinary open access archive for the deposit and dissemination of scientific research documents, whether they are published or not. The documents may come from teaching and research institutions in France or abroad, or from public or private research centers.
L'archive ouverte pluridisciplinaire HAL, est destinée au dépôt et à la diffusion de documents scientifiques de niveau recherche, publiés ou non, émanant des établissements d'enseignement et de recherche français ou étrangers, des laboratoires publics ou privés. 


\title{
Effet d'une levée de dormance incomplète sur la qualité des étamines et du pollen chez le frai- sier (Fragaria $\times$ ananassa Duch)
}

\author{
Georgette RISSER
}

I.N.R.A., Station d'Amélioration des Plantes maraîchères, Centre de Recherches d'Avignon, F 84140 Montfavet

\begin{abstract}
Chez la variété de fraisier « Belrubi », l'observation des étamines et du pollen sur des plants cultivés en serre à partir de 4 dates, 13 octobre, 15 novembre, 5 janvier, 28 février, montre que, pour une même date d'observation, les fleurs des plants des 2 premières séries présentent des étamines moins bien formées et un pourcentage plus élevé de grains de pollen avortés que les fleurs des plants des séries entrées en serre plus tardivement. Cultivées en serre à partir du 13 octobre, 2 variétés précoces à faible dormance " Favette " et "Gariguette " forment des étamines et du pollen de bonne qualité.

La malformation des étamines et l'avortement du pollen ainsi constatés chez «Belrubi » sont attribués à une
\end{abstract} mauvaise levée de dormance.

Mots clés additionnels : Avortement du pollen, malformation des étamines.

Influence of incomplete dormancy breakage on stamen and pollen quality in strawberry (Fragaria $\times$ ananassa Duch).

Plants of the strawberry cultivar "Belrubi" were grown in the glasshouse from October 13, November 15, January 5, and February 28 . At flowering, stamens and pollen were observed. Observed at the same time, stamens were less developed and percentage of aborted pollen grains was greater on the plants moved to the glasshouse on October 13 and November 15 than on the others. Two early cultivars with light dormancy were also grown in the glasshouse since October 13 and had better stamens and pollen than "Belrubi" plants. This anther and pollen failure of "Belrubi" flowers was attributed to bad dormancy breakage.

Additional key words : Pollen abortion, stamen underdevelopment.

\section{INTRODUCTION}

Dans les cultures précoces de fraisiers, on observe souvent la présence de fruits déformés, conséquence d'une mauvaise fécondation (KRONENBERG, 1959. D’ERCOLE, 1979 ; LEMAITRE, 1978 ; I.T.A.P.I., 1981).

Cette mauvaise fécondation peut avoir des causes multiples mais, le plus souvent, dans les conditions de culture française, elle est due à une insuffisance pollinique liée à un avortement partiel des étamines.

L'influence de très nombreux facteurs tels que la variété (KRONENBERG, 1959) voire le clone (KRONENBERG et al., 1959), les températures (WAY, 1967; SAITO \& UENO, 1972), les jours courts (HEIN, 1953), la luminosité (SMEETS, 1976, 1980), l'alimentation minérale (GUTTRIDGE \& ANDERSON, 1974; GUTTRIDGE, 1979 ; KATO \& YAMAZAKI, 1979), certains fongicides, a déjà été montrée mais, jusqu'ici, la dormance n'avait pas été mise en cause. Or, d'une série d'essais effectués pendant l'hiver 1983-1984 à la Station d'Amélioration des Plantes maraîchères de l'I.N.R.A. à Montfavet, il ressort qu'une levée de dormance incomplète entraîne un développement anormal des étamines et du pollen.

\section{MATÉRIEL ET MÉTHODES}

\section{A. Matériel végétal}

Des plants de la variété «Belrubi » (obtention I.N.R.A.) prélevés dans la pépinière de multiplication de la Station d'Amélioration des Plantes maraîchères de l'I.N.R.A. à Montfavet, ont été arrachés, empotés et mis en serre à 4 dates successives : série 1:13 octo- 
bre, série $2: 15$ novembre, série $3: 5$ janvier, série 4 : 28 février. 16, 12, 10 et 10 plantes ont été observées respectivement dans les séries $1,2,3$ et 4 .

Trente deux plantes de "Favette " et 24 plantes de "Gariguette », variétés précoces à faible dormance (obtention I.N.R.A.) ont également été observées dans les conditions de la série 1 .

Les plantes étaient cultivées en pots, en serre verre chauffée (température de nuit : $17^{\circ}$ à $20^{\circ} \mathrm{C}$, de jour : $22^{\circ}$ à $30^{\circ} \mathrm{C}$ ), sans éclairage d'appoint direct (l'éclairage d'autres compartiments de serre a pu toutefois avoir un certain effet).

\section{B. Méthodes d'observation}

Les fleurs sont observées le jour de leur épanouissement. On note le développement des étamines de 0 (absence d'étamines) à 5 (belles étamines à anthères bien gonflées). Sur certaines fleurs, un examen microscopique du pollen est effectué : 6 étamines sont écrasées dans un peu de carmin acétique et au moins 20 grains de pollen dans au moins 5 champs microscopiques (soit un minimum de 100 grains par fleur) sont observés et classés en 2 catégories : grains avortés ou non avortés (fig. 1).

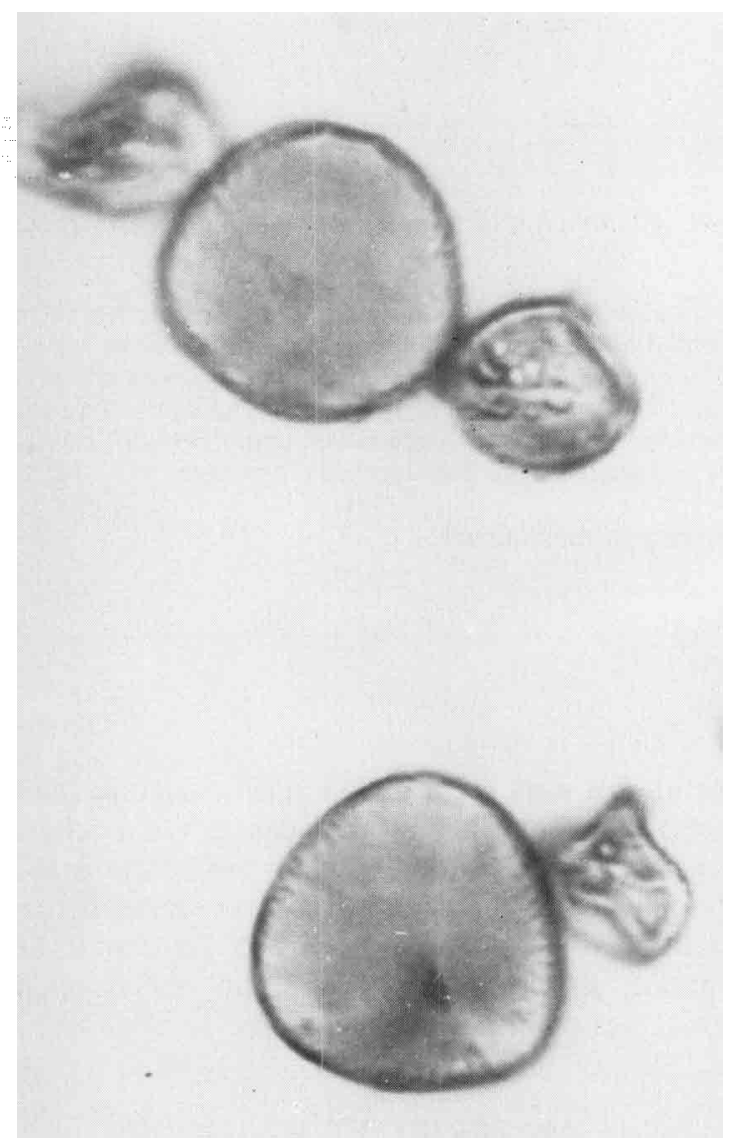

Figure 1

Grains de pollen de « Belrubi » après coloration par le carmin acétique : mélange de grains ronds, bien colorés, d'aspect normal et de grains avortés, déformés, non colorés.

Pollen grains from "Belrubi" after aceto-carmine staining : mixture of normal, round well coloured grains and of aborted, distorted and uncoloured grains.
L'observation des fleurs de "Belrubi » est fait presque chaque jour du 12 décembre au 4 mars, plus irrégulièrement par la suite. La floraison des séries 1 et 2 est continue du 12 décembre à la fin de l'essai (17 mai) alors que celle des séries 3 et 4 est beaucoup plus limitée dans le temps: la série 3 a fleuri du 29 janvier au 27 mars, la série 4 du 17 mars au 15 avril.

La floraison des plantes de "Favette " est assez courte, du 20 novembre au 16 décembre, et est observée régulièrement ; "Gariguette » fleurissait encore fin février mais son observation n'a pas été poursuivie au-delà du 26 février.

\section{RÉSULTATS}

\section{A. Observations des étamines}

La figure 2 présente la répartition des fleurs de «Belrubi » selon le développement de leurs étamines par série et période de floraison. Par simplification, nous avons regroupé les notes 0 à 2 qui correspondent aux fleurs couramment désignées comme "à mauvaises étamines " et les notes 4 et 5 qui correspondent aux fleurs à «bonnes étamines ». Comme prévu, la qualité des étamines s'améliore quand la longueur du jour augmente mais, ce qui est plus inattendu, on constate que, pour une même période de floraison, les étamines des séries 3 et 4 sont beaucoup mieux développées que celles des séries 1 et 2 .

Les variétés 《Favette» et «Gariguette » rentrées en serre en même temps que "Belrubi » (série 1) présentent des étamines de bien meilleure qualité (tabl. 1).

\section{B. Observation du pollen}

Le pourcentage de pollen d'aspect normal par série, période d'observation et note de développement des étamines pour «Belrubi » est rapporté dans le tableau 2 : pratiquement tout le pollen est avorté dans les étamines notées 1 et 2 (les fleurs n'étant pas ensachées, le faible pourcentage de pollen correct observé peut venir d'autres fleurs); au-delà, le pourcentage de pollen normal s'accroît avec le développement des étamines; pour une même note de développement des étamines le pourcentage de pollen correct est plus grand dans les séries 3 et 4 .

L'observation des seules étamines apparemment normales (4 et 5) pendant la période de floraison commune aux 4 séries montre qu'une forte majorité des fleurs des séries 1 et 2 avait moins de 20 p. 100 de pollen bien coloré (tabl. 3).

Comparées aux fleurs de "Belrubi » de la série 1 , celles de «Favette » et de " Gariguette » ont un pourcentage de grains de pollen bien colorés supérieur (tabl. 4).

\section{DISCUSSION}

Ainsi, pendant la période de floraison commune (6 mars au 8 avril), où les conditions d'éclairement et de température sont les mêmes pour les 4 séries, des 


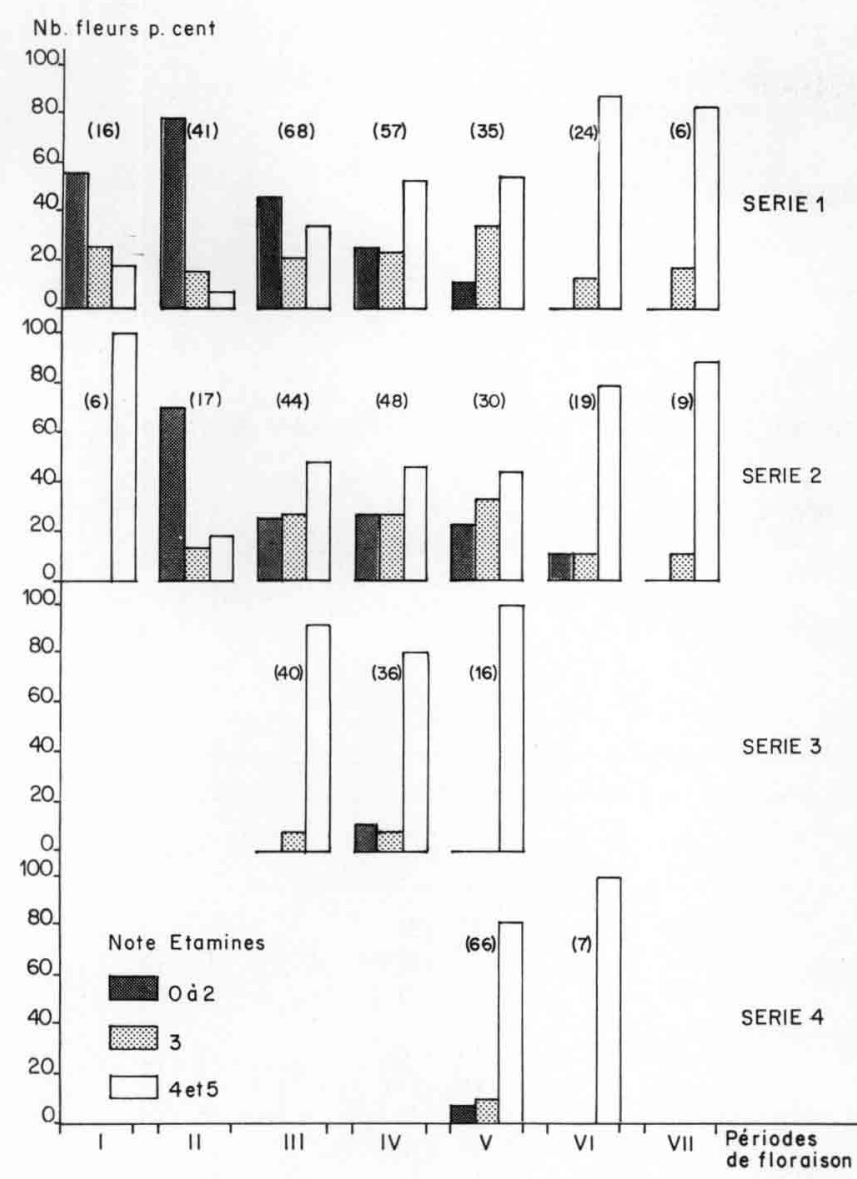

Figure 2

Répartition des fleurs de "Belrubi 》 (en pourcentage) en fonction du développement de leurs étamines, par série et périodes de floraison. Le nombre de fleurs observées figurent entre parenthèses.

Période de floraison Série

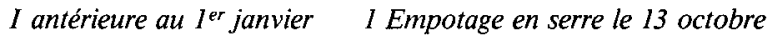

II du ler au 20 janvier $\quad 2 \quad-\quad-\quad 15$ novembre

III du 21 janvier au 12 février $3 \quad-\quad-\quad 5$ janvier

IV du 13 février au 5 mars $4 \quad \ldots \quad$ - 28 février

$V$ du 6 mars au 8 avril

VI du 9 au 29 avril

VII postérieure au 29 avril

Histogram of flowers of "Belrubi" (percentage) according to their stamen development for each series and flowering time. Observed flower number in brackets.

Blooming period

I before January I

II from January I to January 20

III from January 21 to February 12

IV from February 13 to March 5

$\checkmark$ from March 6 to April 8

VI from April 9 to April 29

VII after April 29

\section{Series}

I plants potted in greenhouse on October 13

2 - $-\quad$ - $\quad$ November 15

3 - - $\quad-\quad$ January 5

$4-\quad-\quad-\quad$ February 28

TABLEAU 1

Qualité comparée des étamines chez 3 variétés empotées en serre le 13 octobre (les effectifs de fleurs observées figurent entre parenthèses). Stamen quality for 3 cultivars, potted in the glasshouse on October 13 (number of flowers observed between brackets).

\begin{tabular}{|c|c|c|c|}
\hline \multirow{2}{*}{ Période de floraison } & \multicolumn{3}{|c|}{ Valeur moyenne des étamines } \\
\hline & Belrubi S1 & Favette & Gariguette \\
\hline du 23 novembre au 4 janvier & & $4,75 \quad(67)$ & $3,3 \quad(23)$ \\
\hline du 5 au 18 janvier & $2,5 \quad(2)$ & $4,47 \quad(101)$ & 3,5 (52) \\
\hline du 19 décembre au $1^{\text {er }}$ janvier & $2,0 \quad$ (14) & & $3,8 \quad(56)$ \\
\hline du 2 au 15 janvier & $1,8 \quad(30)$ & & $4,2 \quad(50)$ \\
\hline du 16 au 29 janvier & $2,4 \quad(28)$ & & $4,5 \quad(45)$ \\
\hline du 30 janvier au 12 février & $2,7 \quad(51)$ & & $4,1 \quad(38)$ \\
\hline du 13 au 26 février & $3,1 \quad(39)$ & & $3,8 \quad(30)$ \\
\hline
\end{tabular}

phénomènes d'avortement des étamines et du pollen se sont manifestés chez « Belrubi » beaucoup plus gravement chez les séries 1 et 2 que chez les séries 3 et 4 . Les séries 1 et 2 diffèrent également morphologiquement des séries 3 et 4 , leurs pétioles et leurs hampes florales sont notamment beaucoup plus courts, raccourcissement caractéristique de l'état dormant chez le fraisier. Chez cette espèce en effet, la dormance induite par les conditions automnales n'est pas complète et ne se manifeste pas par un arrêt total de la croissance mais par une croissance ralentie et anormale (fig. 3). Les plantes des séries 3 et 4 transplantées en serre alors que la dormance a été levée par les températures froides hivernales présentent un aspect normal. Ces mêmes températures froides ont interrompu l'initiation florale d'où la courte durée de la floraison de ces séries, contrairement à celles des séries 1 et 2 chez lesquelles une initiation florale ininterrompue a entraîné une floraison continue.

Le moins bon développement des étamines et la moindre proportion de grains de pollen bien colorés chez les plantes des séries 1 et 2 paraissent donc liés à l'état dormant des plantes de ces 2 séries. Une mauvaise levée de dormance pourrait expliquer les fréquentes anomalies de pollinisation observées chez certaines variétés dans les zones méditerranéennes. 
TABLEAU 2

Pourcentage moyen de pollen bien coloré en fonction du développement des étamines chez «Belrubi » (les effectifs de fleurs observées figurent entre parenthèses).

Période de floraison

$I$ antérieure au $l^{\text {er }}$ janvier

II du lor au 20 janvier

III du 21 janvier au 12 février

IV du 13 février au 5 mars

$V$ du 6 mars au 8 avril

VI du 9 au 29 avril

VII postérieure au 29 avril
Série

1 Empotage en serre le 13 octobre

$2 \quad-\quad-\quad 15$ novembre

$4 \quad-\quad-\quad \begin{array}{r}58 \text { féviler } \\ 4\end{array}$

Percentage of well coloured pollen grains according to stamen development for "Belrubi" (observed flower number in brackets).

Flowering period

$I$ before January $I$

II from January 1 to January 20

III from January 21 to February 12

IV from February 13 to March 5

$\checkmark$ from March 6 to April 8

VI from April 9 to April 29

VII after April 29

\begin{tabular}{|c|c|c|c|c|c|c|c|c|c|c|c|}
\hline \multirow{4}{*}{$\begin{array}{c}\begin{array}{c}\text { Période } \\
\text { de } \\
\text { floraison }\end{array} \\
\text { I }\end{array}$} & \multirow{3}{*}{ Série } & \multicolumn{10}{|c|}{ Note de développement des étamines } \\
\hline & & \multicolumn{2}{|c|}{1} & \multicolumn{2}{|c|}{2} & \multicolumn{2}{|c|}{3} & \multicolumn{2}{|c|}{4} & \multicolumn{2}{|c|}{5} \\
\hline & & 5 & (3) & - & - & 13 & (3) & 32 & (3) & - & - \\
\hline & $\mathrm{S} 2$ & & & & & - & - & 15 & (1) & 32 & (3) \\
\hline \multirow[t]{2}{*}{ II } & $\mathrm{S} 1$ & 0 & (5) & 3 & (4) & 11 & $(2)$ & 22 & (2) & - & - \\
\hline & S2 & 0 & (1) & 12 & (1) & & & & & & \\
\hline \multirow[t]{3}{*}{ III } & $\mathrm{S} 1$ & 0 & (4) & 4 & (8) & 7 & $(8)$ & 19 & (10) & 19 & $(6)$ \\
\hline & $\mathrm{S} 2$ & 3 & (2) & 0 & (2) & 11 & (6) & 18 & (15) & 27 & (2) \\
\hline & S3 & - & - & - & - & 26 & (1) & 25 & (3) & 37 & (17) \\
\hline \multirow[t]{3}{*}{ IV } & $\mathrm{S} 1$ & 0 & $(2)$ & 1 & (3) & 10 & (3) & 19 & (14) & 42 & (5) \\
\hline & $\mathrm{S} 2$ & 0 & (4) & 5 & (3) & 11 & (7) & 16 & (7) & 36 & (2) \\
\hline & S3 & - & - & 1 & (3) & - & - & 43 & (10) & 40 & (6) \\
\hline \multirow[t]{4}{*}{ V } & $\mathrm{S} 1$ & - & - & 2 & (2) & 6 & $(8)$ & 8 & $(10)$ & 35 & (5) \\
\hline & $\mathrm{S} 2$ & - & - & 2 & (6) & 5 & (4) & 17 & (7) & 26 & (2) \\
\hline & S3 & - & - & - & - & - & - & 23 & (4) & 52 & (8) \\
\hline & $\mathrm{S} 4$ & - & - & 7 & (1) & 33 & $(2)$ & 20 & (4) & 55 & (19) \\
\hline \multirow[t]{3}{*}{ VI } & $\mathrm{S} 1$ & - & - & - & - & 4 & $(2)$ & 20 & (8) & 53 & (5) \\
\hline & S2 & - & - & - & - & 3 & (1) & 18 & (11) & 36 & (2) \\
\hline & S4 & & & & & & & 94 & (1) & 64 & (5) \\
\hline \multirow[t]{2}{*}{ VII } & $\mathrm{S} 1$ & - & - & - & - & 35 & (1) & 34 & (3) & 68 & (2) \\
\hline & $\mathrm{S} 2$ & - & - & - & - & 2 & (1) & 26 & (8) & - & - \\
\hline
\end{tabular}

TABLEAU 3

Répartition (effectif) des fleurs à étamines « normales» (notes 4 et 5) selon leur pourcentage de pollen bien coloré; période d'observation : 6 mars-16 avril ; variété « Belrubi ».

Number of flowers with good stamens (grade 4 and 5) according to the percentage of well coloured pollen grains; observation period: from March 6 to April 16; cultivar "Belrubi".

\begin{tabular}{|c|c|c|c|c|c|c|}
\hline & \multicolumn{5}{|c|}{ Pourcentage de pollen bien coloré } & \multirow{2}{*}{$\begin{array}{c}\text { Effectif } \\
\text { total }\end{array}$} \\
\hline & $0-5$ & $6-20$ & $21-40$ & $41-60$ & 60 & \\
\hline Série 1 & 5 & 6 & 5 & 2 & 0 & 18 \\
\hline Série 2 & 3 & 6 & 3 & 0 & 0 & 12 \\
\hline Série 3 & 2 & 1 & 1 & 4 & 4 & 12 \\
\hline Série 4 & 1 & 1 & 7 & 8 & 10 & 27 \\
\hline
\end{tabular}

Une confirmation de l'importance des phénomènes de dormance sur la qualité des étamines nous est fournie par les différences de comportement variétal.

Nous voyons que pour une même période, les étamines sont beaucoup mieux développées chez " Favette » et « Gariguette » que chez « Belrubi ». La qualité des étamines est particulièrement bonne chez « Favette " dont la végétation ne présente aucun signe de dormance. Il est également à remarquer que chez cette variété la période de floraison est très courte. La valeur du pollen de «Favette », par contre, est inférieure à celle de "Gariguette "; indépendamment des 
TABLEAU 4

Qualité comparée du pollen chez 3 variétés empotées en serre le 13 octobre (les effectifs de fleurs observées figurent entre parenthèses). Pollen quality for 3 cultivars, potted in the glasshouse on October 13 (observed flower number in brackets).

\begin{tabular}{|c|c|c|c|}
\hline \multirow{2}{*}{ Période de floraison } & \multicolumn{3}{|c|}{$\begin{array}{l}\text { Pourcentage moyen de pollen bien coloré } \\
\text { dans les étamines normales ( } n: 4 \text { et } 5 \text { ) }\end{array}$} \\
\hline & Belrubi SI & Favette & Gariguette \\
\hline du 23 novembre au 4 janvier & & $59 \quad(32)$ & $79 \quad(3)$ \\
\hline du 5 au 18 janvier & 25 (1) & $52(68)$ & $76 \quad(16)$ \\
\hline du 19 décembre au $1^{\text {er }}$ janvier & $36 \quad(2)$ & & $71 \quad(12)$ \\
\hline du 2 au 15 janvier & $22(5)$ & & 53 (4) \\
\hline du 16 au 29 janvier & $17 \quad(6)$ & & $77 \quad(15)$ \\
\hline du 30 janvier au 12 février & $15(10)$ & & $79 \quad(6)$ \\
\hline du 13 au 26 février & $25 \quad(14)$ & & $83 \quad(26)$ \\
\hline
\end{tabular}

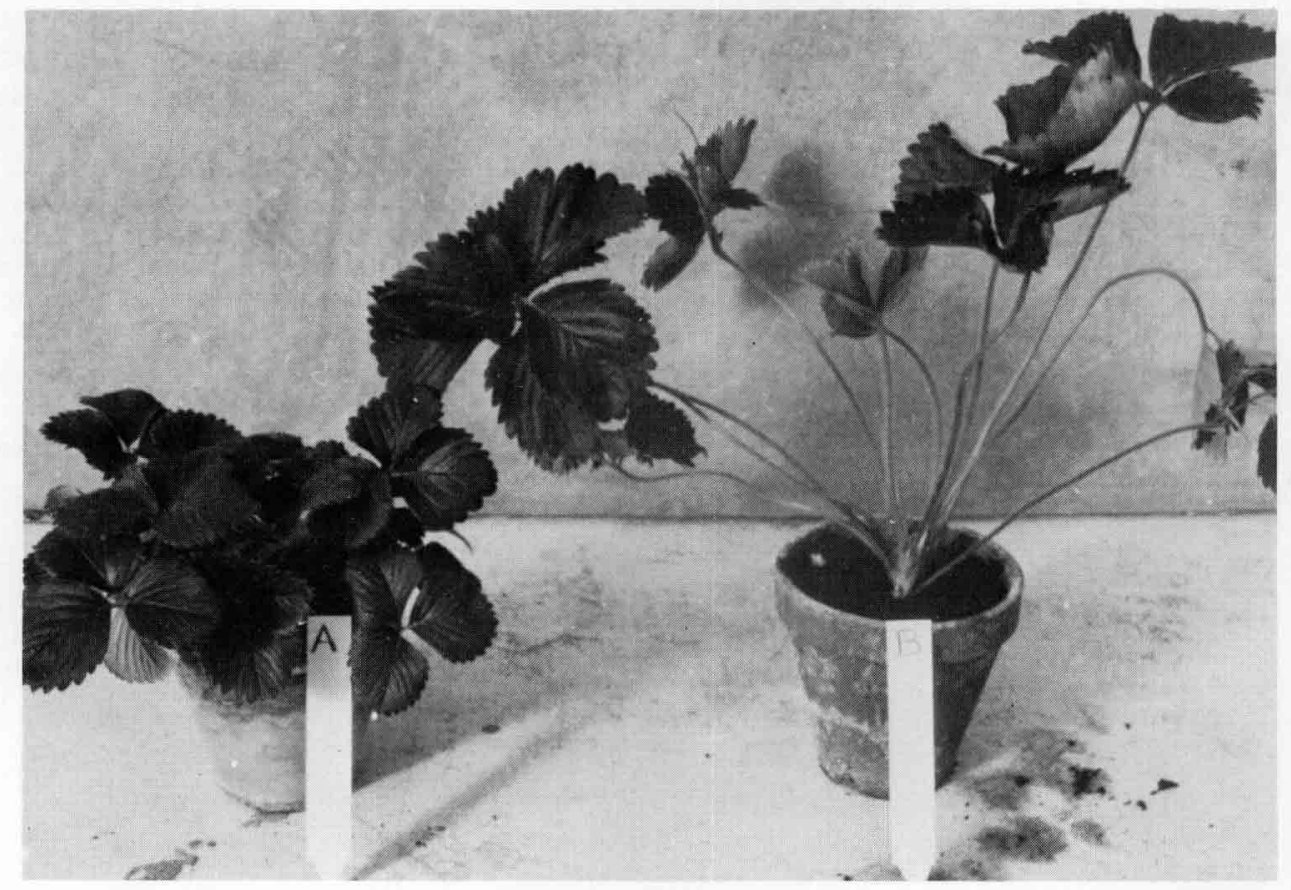

Figure 3

Plants de "Belrubi 》 le 23 mars

$A$ : plant de série 2

$B$ : plant de série 3.
Plants of "Belrubi" on March 23

$A$ : series 2

$B$ : series 3. grains avortés, on observe chez cette variété une grande fluctuation de grosseur des grains de pollen bien colorés. Chez le fraisier, octoploïde de structure ordinairement très hétérozygote, il est fréquent, chez certaines variétés, de trouver une proportion plus ou moins forte de pollen avorté même dans des étamines d'apparence très normale dans des conditions d'environnement satisfaisantes (KRONENBERG, 1959 ; TOMBESI, 1964).

Le cycle physiologique complexe du fraisier est sans doute contrôlé par un équilibre hormonal dépendant des conditions de milieu (GuTTRIDGE, 1969). A des substances de type gibbérelline sont attribués les effets de croissance active et d'inhibition de l'initiation flo- rale (GUTTRIDGE, 1960 ; PORLINGIS \& BOYNTON, 1961 ; LESHEM \& KOLLER, 1966 ; TANABE et al., 1976). MikHTEleVA \& PETRovSKaya-Baranova, en 1974, ont montré que l'acide gibbérellique favorisait le développement des anthères et PARLimAN et al. (1972) ont obtenu des stérilités mâles par traitement de fraisiers avec de l'ethephon, produit considéré généralement comme «anti-gibbérelline ». Il est donc vraisemblable que le développement des étamines est sous l'influence de la même hormone qui favorise le développement végétatif et inhibe l'induction florale. 


\section{RÉFÉRENCES BIBLIOGRAPHIQUES}

D'Ercole N., 1970. Anomalie di sviluppo del frutto della fragola. Atti del $4^{\circ}$ convegno nazionale della fragola, Cesena (Italia), 2123 Maggio, 297-301.

Guttridge C. G., 1960. The physiology of flower formation and vegetative growth in the strawberry. Bull. Inst. Agron. Stn. Rech., Gembloux (Belgique), Hors-série, vol. II, 941-948.

Guttridge C. G., 1969. Hormone physiology of growth regulation in strawberry. S.C.I. Monogr. $n^{\circ} 31$ « Plant growth regulators », 157 169.

Guttridge C. G., 1979. Anther failure main cause of poor fruit set in Redgauntlet. Grower, 91 (11), 38-39.

Guttridge C. G., Anderson H. M., 1974. Fruit malformation in strawberries : can it be avoided ? Grower, 81 (19), 921-922.

Hein G., 1953. Abnormalities in the flower development of strawberries resulting from short day treatments. Meded. Dir. Tuinb., 16, 269-271.

I.T.A.P.I. (Institut Technique de I'Apiculture), 1981. La pollinisation du fraisier. Bull. Tech. Apic., 8 (3), 187-194.

Kato T., Yamazaki H., 1979. Studies on abnormal flower development in greenhouse strawberries. II. Effect of fertilizer control after transplanting. Agriculture and Horticulture (Nogyo oyobi Engei), 54 (7), 921-922.

Kronenberg H. G., 1959. Poor fruit setting in strawberries. I. Causes of a poor set in strawberries in general. Euphytica, 8, 47-57.

Kronenberg H. G., Braak J. P., Zeiliinga A. E., 1959. Poor setting in strawberries. II. Malformed fruits in Jucunda. Euphytica, 8, 245251.

Lemaître R., 1978. Good pollination = good harvest. Fruit belge, 46, 89-91.

Leshem Y., Koller D., 1966. The control of flowering in the straw- berry Fragaria ananassa Duch. II. The role of gibberellins. Ann. Bot. Lond., 30, 587-595.

Mikhteleva L. A., Petrovskaya-Baranova T., 1974. The effect of gibberellin on the differentiation of strawberry stamens and pistils. In Fitogormony v Protsessakh Rosta i Razvitiya Rastenii, Moscow, USSR, Nauka, 42-53 (Ru) from Referativnyi Zhurnal (1975), 5.55.850.

Parliman B. J., Ahn S. H., Harris G., 1972. Induction of male sterility in fruit crops with ethephon. Hort. Science, 7 (3), 322 (Abstr. 48).

Porlingis I. C., Boynton D., 1961. Growth responses of the strawberry plant to gibberellic acid and to environmental conditions. Proc. Am. Hortic. Sci., 78, 261-269.

Saito T., Ueno R., 1972. Studies on the culture of strawberries in plastic houses in an area of snow, low temperature and little sunshine. I. The occurence of malformed fruits. Bull. Shimane Agric. Exper. Stn., 10, 83-96 (Ja).

Smeets L., 1976. Effect of light intensity on stamen development in the strawberry cultivar "Glasa". Scientia Hortic., 4, 255-260.

Smeets L., 1980. Effect of the light intensity during flowering on stamen development in the strawberry cultivars Karina and Sivetta. Scientia Hortic., 12 (4), 343-347.

Tanabe K., Hayashi S., Hirata N., Yamamoto Y., 1976. Physiological studies on dormancy of strawberry (Fragaria grandiflora $\mathrm{cv}$. Hokowase). I. Growth habit in the period of dormancy and changes of growth regulating substances in the crown with progress of dormancy. Bull. Fac. Agric. Tottori Agric., 28, 1-9.

Tombesi A., 1964. La qualita del polline di 4 cultivars di fragola. Italia agric., $\mathrm{n}^{\circ}$ spécial « La Fragola », 7, 767.

Way D. W., 1967. Strawberry fruit malformation. Rep. E. Malling Res. Stn. for 1967, 199-205. 